\title{
La culture technique et le monde contemporain
}

\section{Technological culture and the contemporary world}

\author{
Bruno Jacomy ${ }^{1}$ \\ ${ }^{1}$ Conservateur en chef honoraire du patrimoine, ancien directeur scientifique du Musée des Confluences, Lyon, \\ bruno.jacomy@free.fr
}

\begin{abstract}
RÉSUMÉ. Cette contribution s'attache à montrer l'importance de la culture technique à l'heure où le numérique s'insère dans nos habitudes quotidiennes. Elle part du constat que la technologie et l'innovation n'ont pas une place suffisante dans notre culture contemporaine, et notamment dans la formation des techniciens et ingénieurs. Pour pallier cette lacune, l'auteur propose une méthode d'analyse partant de l'objet technique et croisant deux dimensions complémentaires : celle de l'évolution des objets eux-mêmes et celle de la place des innovations dans le temps long de l'histoire. Les quelques exemples proposés visent à illustrer cette innovation en train de se faire et les moyens de l'enrichir.

ABSTRACT. This contribution seeks to show the importance of technical culture at a time when digital technology is part of our daily habits. It starts from the observation that technology and innovation do not have a sufficient place in our contemporary culture, and in particular in the training of technicians and engineers. To overcome this deficiency, the author suggests a method of analysis based on the technical objects and the crossing of two complementary dimensions: that of the evolution of the objects themselves and that of the place of innovation in the long period of history. The few examples proposed are intended to illustrate this innovation in the making and the ways to enrich it.

MOTS-CLÉS. Numérique, culture technique, approche généalogique de l'objet, évolution, technique et civilisation, société contemporaine, enseignement technique, innovation industrielle.

KEYWORDS. Digital Age, Technical Culture, Genealogical Approach of the Object, Evolution, Technology and Civilization, Contemporary Society, Technical Education, Industrial Innovation.
\end{abstract}

\section{Introduction}

Le Palais de la découverte a fêté ses quatre-vingts ans en 2017 et la Cité des sciences et de l'industrie ses trente ans en 2016. À cette occasion, un colloque ${ }^{1}$ a réuni les principaux acteurs historiques de cette aventure culturelle qui a vu notamment naître, au fil des années, les centres de culture scientifique, technique et industrielle, la fête de la science et nombre de festivals, d'expositions et d'émissions de radio ou de télévision. Plusieurs muséums d'histoire naturelle ont aussi bénéficié d'importantes rénovations, témoignant de l'intérêt des sciences dans notre société et, au-delà, dans la culture contemporaine et le débat public.

On ne peut que se réjouir de voir ainsi mise sur le devant de la scène et au service de tous la « science en train de se faire » [BLA 16], mais en est-il de même pour le monde des techniques ? Bien sûr, le tournant du $\mathrm{XXI}^{\mathrm{e}}$ siècle a vu le Musée des arts et métiers rouvrir ses portes dans une présentation totalement rénovée et ses collections prendre place dans des espaces de conservation d'une qualité jusque-là réservée aux collections de beaux-arts [FER 97]. De même, la Technologie a fait son entrée dans l'enseignement général comme discipline à part entière. Mais, au bout du compte, la technique et l'industrie font-elles pour autant aujourd'hui partie intégrante de notre culture au même titre que les sciences? Rien n'est moins sûr, et les concepts de culture technique ou de culture de l'innovation restent principalement cantonnés, à ce jour, au vocabulaire professionnel. Pourtant, la nécessité de promouvoir une véritable culture technique, dans un contexte où une grande partie des objets que nous utilisons mobilisent des techniques numériques, n'en est que plus indispensable. Je

1 «La muséologie scientifique, toute une histoire! », colloque organisé par Universcience, Paris, 24-25 novembre 2016. 
propose ici de passer au crible de l'actualité ces notions de culture technique et de culture de l'innovation en proposant une esquisse de méthode.

Dans un précédent article [JAC 93], je reprenais à mon compte les définitions de la culture technique telles que les exposait Philippe Roqueplo [ROQ 83] en 1983. Joël Lebeaume [LEB 03] les a repris depuis en comparant les différentes acceptions de « culture technique » et de « technologie » aux «trois facettes qui définissaient au $\mathrm{XIX}^{\mathrm{e}}$ siècle l'éducation intégrale : la main, l'esprit et le cour, en d'autres termes, respectivement, le travailleur, l'homme et le citoyen » (Lebeaume, Vers la technologie et la culture technique pour tous. Quelques repères du passé pour penser les défis de demain, 2003). La main fait référence à cette culture de l'ouvrier, du technicien, de l'ingénieur, qui suppose de maîtriser savoirs et savoir-faire du métier, de connaître la pratique, la technologie professionnelle. L'esprit correspond davantage à une conception humaniste d'hommes et de femmes susceptibles de maîtriser leur environnement technique par le biais des objets qu'ils utilisent. Le cour, enfin, comporte une dimension critique et culturelle plus appuyée : pourquoi met-on en œuvre telle ou telle technique, comment en est-on arrivé là, vers où allons-nous?

\section{Technique et innovation à l'ère du numérique}

Il est toujours difficile de jeter un regard d'historien sur les périodes les plus récentes. Le recul nous manque pour discerner ce qui relève de la suite logique d'événements et ce qu'on peut analyser comme de profonds bouleversements. Il est indéniable - il suffit de regarder nos contemporains dans leur comportement social - que les nouveaux objets et les pratiques que ces derniers nous imposent marquent une rupture avec ce qui était notre environnement quotidien dans un monde industriel hérité de la mécanique, de la machine à vapeur, de l'électricité même. Les changements du dernier demisiècle affectent notre vie, nos rapports humains, nos rythmes journaliers, les distances qui nous séparent les uns des autres. Dans la seconde édition de mon Histoire des techniques, publiée en 2015 [JAC 15], j'ai changé le titre et le contenu de la partie la plus récente, remplaçant l'ancien intitulé, « De la production de masse à la communication », par «L'âge du numérique ». Lors de la première édition, en 1990, les réseaux numériques faisaient seulement leur apparition dans le grand public, avec le RNIS (réseau numérique à intégration de services) et le Minitel. Le micro-ordinateur était déjà en train de conquérir les bureaux, voire les foyers, mais il était illusoire de prévoir alors l'accélération à venir, due à la miniaturisation et à la montée en puissance des microprocesseurs. Changement brutal ou suite logique ? La communication est sans conteste un élément central de notre monde actuel, et le numérique y joue le rôle de secteur clé.

Deux éléments toutefois gardent toute leur actualité. D'une part, la technique est plus que jamais au cœur de notre vie, qu'il s'agisse des derniers outils de communication, des systèmes automatiques de plus en plus présents, des algorithmes, des nanomatériaux, etc. D'autre part, la pluridisciplinarité est un facteur déterminant dans le contrôle de notre avenir. Je réaffirmerais, comme en 1990, que « parmi les grandes tendances qui émergent aujourd'hui, l'effacement des frontières entre domaines du savoir constitue certainement l'une des conditions les plus favorables à l'innovation » (Jacomy, Une histoire des techniques 1990). Mais il ne semble pas que nous soyons mieux armés qu'hier pour maîtriser cette complexité du monde qu'évoquait naguère Edgar Morin. Aujourd'hui encore d'ailleurs, le sociologue et philosophe récidive en appelant de ses vœux « une éducation où serait enseignée la connaissance complexe » pour tracer la voie à une nouvelle civilisation [MOR 16].

De plus en plus souvent émerge le terme d'Anthropocène pour désigner cette nouvelle ère dans laquelle l'influence des humains sur le monde atteint «la même échelle que les fleuves, les inondations, l'érosion ou la biochimie » (Latour, L'Anthropocène et la destruction de l'image du Globe, 2014). Si le terme ne fait pas l'unanimité, il est indéniable que l'empreinte de l'homme sur la planète est un facteur essentiel à prendre en compte dans les enjeux de la société du $3^{\mathrm{e}}$ millénaire. 


\subsection{Innovation et maîtrise de l'avenir}

Chaque grand pas accompli dans l'évolution des techniques et des sciences provoque des changements inévitables dans la manière d'appréhender le monde, de gérer l'inconnu. Les instruments d'optique - lunettes, microscopes, camera obscura - ont joué un rôle majeur dans la révolution de la pensée scientifique au XVII ${ }^{\mathrm{e}}$ siècle, voire dans la représentation picturale. Cette rupture d'équilibre est toujours d'actualité. Le $\mathrm{XX}^{\mathrm{e}}$ siècle se caractérise souvent par une dématérialisation de nombreuses techniques, et les lois régissant les droits de l'inventeur, par exemple, ont dû s'adapter à ces changements. C'est surtout depuis la dernière guerre que la question de la protection de la propriété intellectuelle est devenue plus complexe, en raison notamment de deux domaines en plein développement : d'une part les logiciels informatiques, d'autre part le génie biologique et le vivant en général.

Pour le premier cas, l'innovation est entrée dans une ère d'ouverture inimaginable il y a quelques années, et due en grande partie à l'appétit grandissant de grandes firmes informatiques. Pour affronter le quasi monopole de quelques créateurs de logiciels et de systèmes d'exploitation, plusieurs « inventeurs» ont mis en circulation des logiciels «libres», tel Linus Torvalds avec le système d'exploitation Linux. Une nouvelle forme d'innovation de réseau a ainsi vu le jour, les logiciels étant depuis constamment améliorés par une communauté d'informaticiens amateurs ou professionnels reliés par le Web. Aux États-Unis notamment, nous rappelait récemment Christophe Lécuyer, « les grandes entreprises sont néanmoins de plus en plus concurrencées par de nouveaux acteurs de l'innovation, les universités et les start-up » (Lécuyer, Manager l'innovation, 2015).

Pour le second cas, peut-on parler d'ouverture, lorsque la Cour suprême des États-Unis a accepté, par un arrêt du 16 juin 1980, que soient brevetées des bactéries génétiquement modifiées ? Mettant le vivant sur le même plan que tout produit de l'ingéniosité humaine, le législateur américain a posé un nouveau problème de société pour lequel les comités d'éthique des différents pays ont dû statuer afin d'établir de nouvelles bornes à la brevetabilité de l'innovation.

Rien n'est jamais acquis en matière d'innovation et de protection de la création, quelle qu'elle soit. Promulguer des lois pour protéger l'inventeur est une des actions indispensables pour promouvoir l'innovation, mais trop légiférer risque de fermer la porte à des inventeurs potentiels ou entrer en conflit avec des règles morales de vie en société et de respect des autres pays.

Nous atteignons ici les limites de compétences de l'historien ou de l'ingénieur. D'aucuns lisent dans le poids grandissant des protections juridiques une mort annoncée de l'esprit d'innovation, voire la « fin de l'avenir » [GIM 92]. D'autres, au contraire, pensent que l'homme trouvera toujours les ressorts nécessaires pour imaginer de nouvelles solutions afin de résoudre les problèmes qui obscurcissent l'avenir de la planète.

\subsection{Technique, innovation et culture}

Le terme de « technique » peut s'appliquer à bien des secteurs, comme ceux relevant du commerce, des arts plastiques ou du sport. Mais nous l'entendons ici en tant qu'ensemble des procédés et des méthodes employés à la production d'objets techniques. Nous parlerons de même ici avant tout de l'innovation technique. Toutefois, si l'on veut s'intéresser à l'innovation comme élément de notre culture, il est nécessaire de penser cette démarche comme une aspiration quasi universelle de l'homme vers la nouveauté, qui le différencie nettement des autres êtres vivants et qui prend sa source aux origines mêmes d'Homo sapiens, avec les premiers outils volontaires.

Plus précisément, dans le domaine des machines et des objets techniques en général, l'innovation désigne en fait deux notions distinctes : d'abord un processus, ensuite son résultat. L'innovation est la 
démarche qui va conduire un individu ou un groupe à mettre au point un objet ou un concept nouveau. Le terme désigne également, dans le langage courant, le résultat même de ce travail de conception : on parle des grandes innovations que sont la machine à vapeur, l'électricité nucléaire ou le GPS. Par rapport à l'invention, on réserve généralement le terme d'innovation à une invention qui a réussi. L'invention est l'acte créatif par lequel une idée prend la forme d'un objet réel, l'innovation y ajoute le caractère social, dû à sa diffusion sous forme d'un produit dans la société.

Culture technique, culture d'innovation, ces deux notions sont, sinon les seules, du moins deux facteurs essentiels pour la maitrise de ces bouleversements qui affectent non seulement le monde occidental, mais l'ensemble des sociétés humaines. Ils constituent un véritable enjeu culturel de civilisation.

\section{Une approche de l'innovation en marche}

\subsection{Une analyse à focale variable}

De nombreux auteurs - sociologues, philosophes, historiens... - se sont penchés sur ce processus de l'innovation en marche. Mais ces réflexions ont généralement bien du mal à parvenir au niveau de l'étudiant, du technicien, et bien évidemment du simple citoyen. Pour appréhender ce processus complexe de l'innovation, qui met en cause non seulement des éléments techniques, mais aussi l'environnement scientifique, social ou juridique, il est indispensable de ne jamais perdre de vue le concept de «système technique » décrit par Bertrand Gille [GIL 78]. C'est dans cet esprit qu'ont été mis en place, notamment à l'Université de technologie de Compiègne, des enseignements où deux dimensions complémentaires ont été mises à contribution pour appréhender le plus finement possible le processus de l'innovation et le mettre en pratique dans une démarche professionnelle. Pour cela, appliquer le raisonnement à un objet précis offre l'opportunité de ne pas quitter la réalité technique tout en questionnant son environnement. Abraham Moles, dans son introduction à la Théorie des objets, en fait le :

«médiateur universel, révélateur de la Société dans la dénaturation progressive de celleci, constructeur de l'environnement quotidien, système de communication sociale, plus chargé de valeurs qu'il ne le fut jamais par le passé, en dépit de l'anonymat de la fabrication industrielle» (Moles, Théorie des objets, 1972).

Suivons donc A. Moles dans sa proposition, prenons l'objet comme marqueur de cette évolution et, pour bien comprendre comment les choses changent, munissons-nous, virtuellement bien sûr, d'instruments d'observation dotés de focales différentes : un grand angle pour appréhender, sur le temps long, les conséquences ou causes de ces micro-évolutions dans la société des hommes, et un objectif macro pour observer à un moment donné de notre histoire comment et pourquoi évoluent certains objets techniques comme les dérailleurs de vélo, les logiciels informatiques ou les scanners.

Si je m'arrête quelques instants sur le "point de vue de Sirius », je m'aperçois que les grands bouleversements techniques, par exemple, affectent et sont induits généralement par un ensemble de secteurs clés tels que l'énergie, les matériaux et la communication. À l'orée de notre histoire, ce furent la domestication du cheval ou du bœuf pour l'énergie, la céramique pour les matériaux et l'écriture pour la communication. À la fin du Moyen Âge, ce furent le moulin, le fer et l'imprimerie. Aujourd'hui, bien malin qui pourra identifier les grands bouleversements à venir. Le numérique, avec ses différentes déclinaisons telles que l'Internet mobile ou les objets connectés, apparaît comme le changement majeur, mais les domaines des bio- ou nanomatériaux, comme celui des cellules solaires souples, sont peut-être porteurs des plus grandes révolutions à venir. 
Les traces matérielles que laissent les civilisations ne sont généralement pas ce qui revêt la plus grande importance pour leurs contemporains. Ce qui va permettre à un objet de survivre aux mortels, ce sont soit ses matériaux résistants (béton, bronze, verre...), soit son usage symbolique ou cultuel (reliques, temples...), soit des conditions climatiques ou physiques particulières (pergélisol, déserts chauds...), soit encore un usage ininterrompu (ustensiles de cuisine, vêtements...). Mis à part, le cas échéant, ceux dont la fonction est esthétique ou rituelle, tout objet peut être considéré comme un objet technique. Il a été conçu et fabriqué avec un authentique savoir-faire technique, dans une tradition qui révèle l'« état de l'art » de son époque. Rappelons-nous cette phrase de Gilbert Simondon à propos de l'aiguille anglaise du XVIII ${ }^{\mathrm{e}}$ siècle :

« Il ne serait pas exagéré de dire que la qualité d'une simple aiguille exprime le degré de perfection de l'industrie d'une nation. [...] Les ensembles techniques s'expriment dans les plus simples éléments qu'ils produisent. » (Simondon, Du Mode d'existence des objets techniques, 1989).

Les objets techniques, quels qu'ils soient, sont les indices nous permettant de poursuivre notre enquête, dans un objectif de connaissance historique ou dans l'optique d'une démarche d'innovation. L'aiguille anglaise de Simondon renvoie à ces deux dimensions. Dans une dimension " macro », le simple état de perfectionnement de ce minuscule objet - la nuance de son acier, sa technique de fabrication - est révélateur du système technique de l'Angleterre en marche vers la révolution industrielle, bien en avance sur les autres pays européens de la fin du XVIII ${ }^{\mathrm{e}}$ siècle. Dans sa dimension « micro », l'objet s'inscrit dans sa généalogie propre, entre de grossières aiguilles de fer, descendantes des clous forgés plus anciens, et de futures aiguilles plus fines, moins agressives pour le fil, annonçant les aiguilles de phonographes à venir un siècle plus tard ou les fines aiguilles inoxydables des chirurgiens.

Les objets techniques sont d'inestimables «pièces à conviction» pour qui est avide de connaissance. Ils ont été, à côté des "merveilles de la nature », parmi les premières "curiosités » collectées et présentées dans les cabinets des $\mathrm{XVII}^{\mathrm{e}}$ et $\mathrm{XVIII}{ }^{\mathrm{e}}$ siècles et ont permis, au prix d'inévitables - et souvent fertiles - erreurs, de comprendre que la compétence technique, la connaissance des matériaux, le savoir pratique étaient partagés par l'humanité toute entière et pas seulement réservés au monde dit civilisé.

Cette curiosité, indispensable au plaisir du savoir, ne peut que s'enrichir d'une bonne pratique de l'observation. On mesure aujourd'hui combien cette dernière qualité mériterait d'être davantage stimulée, mise à profit pour mieux comprendre le monde, son évolution et celle des objets qui nous entourent. Les musées d'art ou d'archéologie recèlent eux aussi des corpus incomparables de découverte. Comment mieux pénétrer le contenu et l'atmosphère d'une forge du $\mathrm{XIX}^{\mathrm{e}}$ siècle que de scruter, par exemple, l'Intérieur de la grande forge de Fourchambault, peint par François Bonhommé en 1839-1840? Et que saurions-nous des techniques de construction du Moyen Âge ou de la Renaissance sans les représentations si détaillées et précises des tableaux de Pieter Bruegel, comme son époustouflante Tour de Babel (vers 1563), dont on peut d'ailleurs aujourd'hui explorer les moindres détails sur Google Arts \& Culture ${ }^{2}$ ?

Je ne sais pas ce que nos élèves en retiendront lorsqu'ils seront adultes, mais on ne peut que se réjouir de voir aujourd'hui inscrites dans les objectifs du cours de Technologie en classe de sixième trois grandes thématiques issues de trois dimensions complémentaires. Pour l'ingénierie : le design, l'innovation, la créativité ; pour la dimension socio-culturelle : les objets techniques, les services et les

2 Voir le tableau du Kunsthistorisches Museum de Vienne en très haute définition à l'adresse http://www.google.com/culturalinstitute/beta/asset/the-tower-of-babel/bAGKOdJfvfAhYQ 
changements induits dans la société ; pour la dimension scientifique : la modélisation et la simulation des objets techniques ${ }^{3}$. Ainsi, les élèves peuvent :

« relier les évolutions technologiques aux inventions et innovations qui marquent des ruptures dans les solutions techniques ; comparer et commenter les évolutions des objets selon différents points de vue : fonctionnel, structurel, environnemental, technique, scientifique, social, historique, économique...» (Ministère de l'Éducation nationale, de l'Enseignement supérieur et de la Recherche, Programmes pour les cycles 2, 3, 4, 2015).

\subsection{Des objets dans leur milieu}

L'histoire des techniques s'est longtemps focalisée sur deux voies souvent présentées comme opposées, ou au mieux complémentaires : une histoire technique des techniques, "internaliste », qui « consiste à mettre en évidence la logique propre de l'évolution des techniques » [DAU 79], et une histoire globale des techniques, qui se propose d'analyser les techniques au sein de systèmes techniques donnés [GIL 78].

Grosso modo, la première voie serait celle des techniciens et la seconde celle des économistes. Dans cette vision simpliste de l'histoire des techniques, où insérer des démarches plus originales comme celle de Gilbert Simondon [SIM 89], qui propose une approche philosophique de l'évolution des systèmes techniques, celle d'un Lynn White, qui part de l'histoire technique de l'étrier pour montrer l'importance que peut revêtir l'évolution d'un simple objet technique dans le développement économique et social de l'Europe médiévale [WHI 69], ou encore celle de David Edgerton, qui plaide pour une « histoire des techniques en usage » fondée davantage sur les objets que sur les techniques [EDG 13]?

Pendant longtemps, en gros pendant la période qui va du milieu du XVII ${ }^{\mathrm{e}}$ siècle au milieu du $\mathrm{XX}^{\mathrm{e}}$, on a fait la distinction entre les sciences, qui nous aident à comprendre le monde, les objets, qui nous permettent d'agir sur la matière ou la mesurer, et les techniques, qui constituent les savoir-faire indispensables pour construire ou manipuler les objets.

Dans le contexte du XIX ${ }^{\mathrm{e}}$ siècle, les distinctions étaient claires. Le moteur à combustion interne, par exemple, est un objet technique - le moteur en lui-même - qui repose sur un principe scientifique - la thermodynamique, les lois de Carnot - et qui utilise des techniques variées - la métallurgie, les systèmes d'étanchéité, la mécanique... Si l'on veut comprendre comment est né le moteur à combustion interne et comment il s'est développé, il est nécessaire de se pencher à la fois sur l'évolution des sciences, sur celle des techniques ou sur celle des objets eux-mêmes. Aujourd'hui, il est souvent bien difficile d'établir des frontières nettes entre ces entités. La neurochirurgie, les nanotechnologies mêlent intimement techniques, sciences et objets, qui eux-mêmes deviennent souvent immatériels, virtuels. De même, si l'on veut imaginer quelles seront les innovations susceptibles de changer notre monde futur, il est indispensable de prendre en compte les différentes facettes de cette évolution.

Le premier moteur à combustion interne, le moteur à gaz pauvre mis au point par Étienne Lenoir en 1861, repose sur la structure de la machine à vapeur mais son principe est résolument nouveau. Les éléments constituant les moteurs à venir varient au cours du temps avec l'évolution des techniques et des matériaux. Leurs performances s'améliorent à la fois par les recherches scientifiques (la compréhension des phénomènes permet d'améliorer le rendement) et par l'émergence de nouvelles techniques (matériaux plus légers, lubrifiants performants qui allongent la durée de vie des moteurs,

3 Annexe 3 Programme d'enseignement du cycle des approfondissements (cycle 4), arrêté du 9-11-2015 - J.O. du 24-112015. 
etc.). Les objets techniques sont ainsi le fruit d'un petit nombre d'innovations majeures et d'une chaîne sans fin d'innovations mineures.

En un sens, les objets évoluent à la manière d'une généalogie familiale. Chaque objet a de multiples ancêtres, dont le mariage était souvent tout à fait fortuit; il suffit de rappeler que la mécanique Jacquard, le tube électronique et la machine à calculer sont tous des ancêtres de nos ordinateurs. En chaque objet est lui-même porteur de descendants tout aussi inattendus.

L'objet technique peut ainsi être considéré comme un « être » en soi, avec sa propre ascendance et sa propre filiation, au sein de ce que Guy Deniélou, président-fondateur de l'Université de technologie de Compiègne, appelait le « règne machinal ». Il nous révèle alors l'histoire des innovations qui se sont succédé et nous permet d'approcher ce cheminement de la pensée technique. Cette histoire se nourrit d'une histoire technique des innovations, indispensable étape d'analyse, mais qui se situe délibérément dans une perspective dynamique, cherchant constamment à s'interroger sur l'enchaînement des innovations et les raisons de tous ordres qui les ont permises.

Dans un second temps, on peut se pencher davantage sur l'utilisateur que sur le créateur. Tout objet est immanquablement inséré dans un système homme-machine qui a son développement intrinsèque. La mécanisation, puis l'automatisation, ont inévitablement des conséquences sur les hommes chargés de mettre en œuvre des techniques données. Cet aspect constitue le cœur d'une histoire sociale largement étudiée par ailleurs. L'originalité de l'approche que j'ai eu l'occasion de développer à propos des riveurs et des machines à river consiste à creuser la question en pénétrant au cœur même des techniques mises en jeu, quitte à démonter le subtil édifice mis en place par les entrepreneurs pour, en général, augmenter la productivité, mais sans oublier les pratiques des ouvriers eux-mêmes, qui font souvent preuve d'une créativité « masquée » [JAC 98].

\section{Innovation et évolution des objets techniques}

Je me hasarderai à une métaphore, pour évoquer le processus qui conduit à l'émergence d'objets nouveaux : la voie urbaine. Nos chaussées sont souvent l'aboutissement d'une stratification, du chemin de terre à la route pavée, puis au macadam et à ces couches successives qu'on observe souvent dans nos villes au hasard des travaux. Et il n'est pas rare de retrouver, sous cette accumulation de matériaux et de techniques, les vestiges d'une voie romaine. Je prends cette métaphore pour illustrer l'évolution graduelle des innovations dans de nombreux domaines. L'automobile, pour prendre un exemple simple, n'a pas fondamentalement évolué dans sa structure depuis les premières voitures de la fin du $\mathrm{XIX}^{\mathrm{e}}$ siècle jusqu'à celles de 2017. Elles ont toujours une carrosserie, quatre roues, un moteur, une transmission et, encore pour quelques années sans doute, un poste de conduite... De même que notre rue est le résultat d'une stratification historique, l'automobile est le fruit d'une lente évolution, marquée par de très nombreux progrès, mais qui n'ont que peu affecté leur structure générale. Si je reviens à mon exemple urbain, que se produit-il lorsqu'une municipalité veut implanter le tramway, situation fréquente ces dernières années ? Dans ce cas, il est indispensable, pour des raisons de charge au sol, de maintenance, de planéité de la voie, d'alimentation électrique, voire de contraintes juridiques, de creuser la rue assez profondément pour dévoyer tous les réseaux existants et rompre avec cette accumulation chronologique de couches. La mise en place de ce nouveau moyen de transport urbain impose alors des perturbations importantes dans la ville : gêne au voisinage et aux commerces, déviations et donc embouteillages, etc. Contrairement à l'évolution graduelle évoquée plus haut, on se trouve ici dans une situation de rupture importante, dont les conséquences affectent profondément le milieu même. Les exemples ne manquent pas de ce double processus. J'ai cité l'automobile comme exemple du premier cas, je citerai la navette volante, la machine à vapeur ou le transistor comme innovations qui ont provoqué d'importants changements dans la société. 


\subsection{Une démarche d'innovation, par petits pas et grands sauts}

Ces deux démarches d'innovation sont bien connues et étudiées par les historiens des techniques, et reprises par les spécialistes en management, comme innovations « incrémentale » et « de rupture ». Elles ne sont toutefois pas forcément partagées par les élèves-ingénieurs, les futurs techniciens ou même les lycéens. Introduire cette connaissance dans les formations techniques ou générales permettrait pourtant de faire entrer une culture de l'innovation dans le processus normal de pensée de ceux qui seront à même de concevoir et fabriquer les objets techniques que nous sommes amenés à utiliser, ainsi que de tous les citoyens qui manipuler au jour le jour ces nouveaux outils.

Ce processus assez général a notamment été étudié assez finement par de nombreux historiens des techniques et des sciences. Ainsi, les écrits classiques de Thomas S. Kuhn [KUH 72] sur les révolutions scientifiques et la science normale mettent en évidence les blocages qui freinent l'émergence de nouveaux paradigmes : des blocages scientifiques, techniques ou parfois psychologiques. Voir aussi les courbes en $\mathrm{S}$ représentant la succession des innovations dans un domaine donné.

Depuis un demi-siècle, l'informatique est sur ce chemin, après la mise au point des grands calculateurs de la Seconde Guerre mondiale et la fixation des principes édictés par John von Neumann et Alan Turing : principe séquentiel, structure binaire et technique électronique. Mais qui aurait pu imaginer, alors que nous assistions ébahis à la grande aventure de la conquête spatiale, que les petitsenfants de cette génération utiliseraient au quotidien, dès leur plus jeune âge, des smartphones dont chacun s'avère plus puissant que toute l'informatique mise en œuvre pour poser un homme sur la Lune?

L'innovation, partout présente, se heurte à de multiples obstacles avant de provoquer des changements importants; des obstacles techniques, scientifiques, mais aussi économiques ou psychologiques. Combien d'années avons-nous dû subir la dictature des écrans cathodiques, archaïques descendants du tube de Crookes de 1869, avant que les écrans à LED n'ouvrent grandes les portes de l'informatique nomade ? Combien de siècles a-t-il fallu pour que le système héliocentrique s'impose à tous ? Pourquoi n'a-t-on pas inventé une machine à calculer dès les débuts de l'horlogerie, lorsqu'on disposait de toutes les techniques et de tous les principes nécessaires ? Il fallait qu'un jeune Auvergnat de 19 ans nommé Blaise Pascal veuille soulager la charge de son père, collecteur des impôts, pour que soit mise au point la première machine à calculer.

Dans le monde des objets, on peut trouver une multitude d'exemples de cette évolution à deux vitesses. Je m'arrêterai un instant sur le cas du sextant. Jusqu'au XVI siècle et depuis l'antiquité, la mesure de la latitude en mer se fait avec des instruments de mesure d'angles assez rudimentaires, tels l'astrolabe de mer ou les divers types de quadrants. L'arbalestrille, ou bâton de Jacob, fut l'instrument le plus perfectionné utilisé lors des grands découvertes de la fin du $\mathrm{XV}^{\mathrm{e}}$ siècle. Depuis l'antiquité, la navigation en haute mer se fait grâce à ces instruments, dont le sablier, hérité lui-même des Égyptiens. Pourtant, en guère plus d'un siècle, entre 1595 et 1730, vont se succéder trois pas importants : le quartier de Davis (peu avant 1600), l'octant de Hadley (vers 1700) et le sextant de Ramsden (vers 1730).

Une fois mis au point, le sextant du XVIII ${ }^{\mathrm{e}}$ siècle ne va pratiquement plus évoluer. Le sextant que nous pouvons acheter aujourd'hui chez un détaillant spécialisé sera identique à celui d'il y a trois siècles, ses matériaux mis à part. En un mot, le sextant est parvenu, dès son apparition, à un stade de maturité technique et fonctionnelle telle que seuls quelques perfectionnements mineurs seront apportés au cours des siècles suivants. 


\subsection{Des familles d'objets pour comprendre l'évolution}

Pour encourager l'innovation, de nombreux facteurs externes ont été utilisés au fil du temps : la protection de la propriété intellectuelle, les facilités économiques, la divulgation des brevets, etc. Mais il est sans doute plus difficile encore de susciter cette innovation en développant l'esprit créatif des hommes. Pour cela, il importe avant tout de comprendre comment, de l'idée à l'objet, le projet se concrétise dans le tête de l'inventeur et celle de l'innovateur. Il est intéressant de prendre alors la loupe et d'observer par exemple, à travers des séries d'objets conservées dans les musées, comment des progrès successifs ont permis de perfectionner les formes, les fonctions, l'ergonomie des objets techniques. Pour prendre un exemple que le public peut découvrir au Musée des Confluences, on peut saisir, à travers la série de microscopes simples de la collection Giordano qui y est présentée, comment on passe, pas à pas, du microscope qu'il faut tenir à la main devant son œil et qui demande un savoirfaire abouti, tels les microscopes d'Antonie van Leeuwenhoek ou de Christiaan Huygens des années 1670, aux microscopes de laboratoire des années 1820 , à la fois performants et d'usage plus pratique. Les méthodes de polissage des lentilles, d'élaboration d'un verre parfait, de réglage micrométrique de la position du spécimen ou la résolution du problème des aberrations chromatiques améliorent sans cesse les performances des nouveaux microscopes. Chaque instrument, chaque partie d'instrument fait l'objet de perfectionnements, souvent minimes, mais dont l'addition fera de l'objet rudimentaire de départ un instrument utilisable à grande échelle, et dont les retombées scientifiques seront considérables. La démarche qui consiste à analyser par le menu les infimes détails qui différencient un appareil de son voisin, fondée avant tout sur l'observation, peut être appliquée à l'étude de tout objet technique. Elle se révèle ainsi un puissant moteur d'analyse pour de futurs techniciens ou ingénieurs.

À la condition de favoriser la double approche évoquée plus haut par des mises en perspective pertinentes, le musée, comme l'école, peuvent nous aider à dégager de ces objets tous les enseignements utiles. De même que, dans les muséums d'histoire naturelle, les espèces sont disposées de manière à faire toucher du doigt l'évolution du monde vivant, les musées techniques ou scientifiques peuvent, par l'exposition de séries cohérentes d'objets manufacturés, permettre à de futurs techniciens ou ingénieurs, comme à de simples curieux, de saisir le cheminement de la pensée technique. Les piles électriques ou les ponts présentés « en famille » au Musée des arts et métiers nous dévoilent, par exemple, les progrès accomplis pas à pas dans les principes chimiques des accumulateurs ou les structures comprimées ou tendues des ponts métalliques.

Depuis l'inventeur qui met au point dans son garage des objets qui, malheureusement, ne franchiront jamais la porte d'un magasin, jusqu'aux usines à inventer tel le laboratoire de Menlo Park créé par Thomas Edison en 1876, le fossé est gigantesque. Et dans le climat de la fin du XIX ${ }^{\mathrm{e}}$ siècle, la question de la rationalisation ne s'applique pas seulement à la production d'objets, mais aussi à la production d'inventions. Comment comprendre le processus de la création, comment l'analyser pour le modéliser et le reproduire à grande échelle ? Il a fallu les travaux de sociologues ou de psychologues au $\mathrm{XX}^{\mathrm{e}}$ siècle pour voir apparaître l'heuristique, la science de l'innovation. La question a été déjà abondamment traitée, et décortiquée notamment par Abraham Moles et René Boirel dans les années 1960, et l'art d'inventer est pratiquement devenu une science en soi : « De science des valeurs, l'heuristique devient une méthodologie de la découverte » [MOL 70] [BOI 72].

Avec le recul du temps, la formalisation de cette analyse détaillée de l'innovation technique met en évidence trois éléments importants pour comprendre l'évolution des techniques et des objets. Primo, les transferts de technologie ne sont pas une invention du $\mathrm{XX}^{\mathrm{e}}$ siècle mais un phénomène constant dans la démarche du progrès technique. De nombreuses innovations majeures sont le fait d'hommes qui ont su, par intérêt personnel ou par une ouverture d'esprit naturelle, récupérer, dans un autre champ de savoir, une solution technique applicable à leur problème. Secundo, l'évolution des objets techniques répond globalement à une série de lois, d'invariants qui permettent de comprendre l'enchaînement des 
innovations et de prévoir les progrès à accomplir. Tertio, cet esprit de création qui anime depuis toujours les hommes a des incidences non seulement sur le progrès technique lui-même, mais également sur l'avancée des sciences et leur impact sur les sociétés. L'idée qui voudrait que les progrès techniques découlent de la science est battue en brèche par d'innombrables exemples. Souvent même, le phénomène est inverse. Ce sont les innovations techniques qui offrent aux savants ou ingénieurs de nouveaux outils ouvrant la voie à des découvertes originales. L'exemple des microscopes simples, cité plus haut, est à cet égard édifiant. C'est le microscope de Leeuwenhoek qui a permis la découverte des micro-organismes, comme la lunette a permis à Galilée de découvrir les satellites de Jupiter. Grâce à ces innovations mises entre les mains d'hommes à l'esprit éclairé, de grands progrès peuvent s'accomplir.

\subsection{Les lois de l'évolution}

Les machines, les objets techniques évoluent, certes, mais de quelle manière ? Y a-t-il des lois qui régissent cette évolution et peut-on prévoir quels seraient les ressorts de ce mouvement ? La question est intéressante d'un point de vue historique, mais elle est capitale pour qui tente de percer les mystères de l'innovation en marche. Invention et évolution technique sont, pour l'ethnologue et préhistorien André Leroi-Gourhan, intimement liées :

«Entre l'invention autonome et l'emprunt pur et simple au voisin, l'écart n'est pas considérable [...]. On n'invente le rouet ou on ne l'emprunte que si l'on est en état de l'utiliser; constatation banale mais qui doit être posée à la base de toute construction d'évolution technique. » (Leroi-Gourhan, Évolution et techniques, 1945).

Depuis Johann von Beckmann à la fin du XVIII ${ }^{\mathrm{e}}$ siècle jusqu'à André-Georges Haudricourt, en passant par Franz Reuleaux et Charles Frémont, nombreux sont les auteurs qui ont tenté de démonter les mécanismes de l'innovation, dans une visée technologique, qu'elle soit industrielle pour la plupart des auteurs les plus anciens, ou qu'elle procède d'une science humaine, comme l'affirment Haudricourt ou François Sigaut [HAU 88][SIG 91]. Chez la plupart des technologues du XIX siècle, ces « ingénieurs penseurs de la technique », la référence à l'évolution du vivant est explicite, mais utilisée comme un moyen d'expliquer l'évolution des objets techniques. Ils appliquent au monde des techniques les méthodes mises au point par les naturalistes et non une « théorie de l'évolution » dans sa globalité. Ce faisant, ils mettent en pratique un principe fondamental, celui de l'analogie, qui fait partie de l'outillage méthodologique de base de l'ingénieur [VER 93]. D'une certaine manière, Gilbert Simondon prolonge cette démarche dans son Mode d'existence des objets techniques : "C'est à partir des critères de la genèse que l'on peut définir l'individualité et la spécificité de l'objet technique.» (Simondon, Du mode d'existence des objtes techniques, 1989). Il dégage pour cela un certain nombre de lois d'évolution des objets techniques, dont le processus de concrétisation - c'est-à-dire l'évolution de l'abstrait vers le concret —, l'autonomie de fonctionnement interne, les tendances vers le simple, le petit, le fermé, etc.

L'apport théorique et méthodologique de Simondon a été propagé par Yves Deforge [DEF 85], qui en a appliqué et vérifié le contenu sur un plus vaste échantillon d'objets, notamment dans sa Technologie et génétique de l'objet industriel ${ }^{4}$. Il y dégage une méthode d'approche génétique des objets techniques, fondée sur les concepts de familles et de lignées, eux-mêmes définis à partir de la fonction d'usage et des principes mis en œuvre dans les objets. Il met aussi en évidence les exceptions et les limites de ces lois, liées par exemple aux caractéristiques des développements récents de notre système économique et industriel. L'apport essentiel de Deforge a été d'en préciser les principes et méthodes à travers un enseignement à l'usage des ingénieurs, conçu comme une formation à la

4 Voir aussi la postface d'Yves Deforge au Mode d'existence de Simondon réédité en 1989. 
démarche créatrice. Le temps long de l'histoire des sociétés peut ainsi expliciter les questions posées par l'analyse du temps court de l'évolution des objets.

\subsection{L'innovation dans l'histoire des hommes}

En observant de loin la Terre et l'histoire des êtres qui la peuplent, on est frappé par deux phénomènes. D'une part, en un lieu donné, des moments particulièrement fertiles en innovations alternent avec des périodes d'accalmie, qui sont aussi en général des temps de développement, d'approfondissement. L'avènement du feu, de l'outil taillé ou de la machine à vapeur correspondent à ces moments d'importante mutation, où des objets ou techniques nouveaux provoquent des bouleversements économiques, sociaux et culturels. D'autre part, sur une échelle géographique plus large, on s'aperçoit que le caractère novateur d'une société va circuler de pays en pays, et s'inscrire à la fois dans l'espace et dans le temps.

Nous savons que diverses espèces animales font preuve d'une véritable propension à résoudre des problèmes liés à leurs besoins vitaux. Ainsi, des primates, ou certains oiseaux, façonnent des outils rudimentaires pour se nourrir ou se protéger. Mais seule l'espèce Homo sapiens va, au cours du temps, s'inscrire dans une démarche permanente de recherche de la nouveauté, de progrès et de transmission de savoir, avec le confort qu'elle peut en retirer mais aussi l'impact inévitable sur son milieu, la planète et les autres espèces vivantes. Créer serait-il le nouveau «propre de l'homme»? En tout cas, les grandes acquisitions techniques de la préhistoire - l'outil et le feu notamment - ont singularisé Homo sapiens comme l'espèce qui va façonner la Terre par ses innovations successives.

En gardant l'objectif réglé sur cette longue histoire, on voit émerger, dans notre vieille Europe, quelques périodes de mutation profonde, qu'on qualifie un peu trop vite parfois de révolutions : le néolithique, l'invention de la mécanique cinq siècles avant notre ère, la "révolution industrielle du Moyen Âge », pour reprendre le terme de Jean Gimpel [GIM 75], la révolution industrielle ou encore les mutations consécutives à l'avènement de l'électricité à la fin du XIX ${ }^{\mathrm{e}}$ siècle. Ces «moments créatifs » correspondent à des périodes où, en raison de conditions climatiques ou économiques favorables, les hommes d'une région apportent des perfectionnements majeurs à une machine ou à une technique - le moulin à eau, la filature, le moteur électrique - qui va provoquer une cascade d'autres innovations, engendrant à leur tour un déséquilibre, une crise, une redistribution des cartes entre les acteurs d'un pays ou entre pays voisins. Ces changements, plus ou moins brutaux, sont suivis de périodes plus longues où un nouvel équilibre va lentement se mettre en place sur ces nouvelles bases.

\section{Conclusion}

Lorsque nos ordinateurs quantiques, alimentés par la fission thermonucléaire, feront pour nous des tâches que nous n'imaginons pas encore aujourd'hui, il est fort probable que nous couperons toujours notre nourriture avec un couteau, cet outil novateur du néolithique composé d'un manche et d'une lame, qui, s'il a changé dans ses matériaux, garde depuis lors une forme et un usage qui n'ont pas varié d'un iota.

Cette permanence est le lien qui nous rattache à notre histoire, mais aussi à nos congénères des cultures des autres pays du monde. Ce qui apparaît évident dans les différents cas, c'est l'impossibilité, à un moment de l'histoire, d'imaginer les innovations majeures à venir.

Pourtant, bien souvent, tout était déjà en place pour que l'innovation ait lieu depuis un moment. C'est aussi pour cela que les résultats des différentes études de prospective récentes, comme celles d'il y a un siècle, sont bien souvent incapables de se projeter dans un avenir un peu lointain. 
Il va de soi que les objets, en eux-mêmes, n'ont pas de mémoire, mais ils portent en eux la mémoire de nos sociétés, ils sont le support de notre propre capacité à dégager des processus d'évolution, des opportunités de progrès, d'innovation. Les auteurs de l'Encyclopédie, au siècle des Lumières, comme les fondateurs de ce grand projet de muséum national universel imaginé sous la Révolution française, avaient bien compris, tel l'abbé Grégoire, que le progrès passait par l'éducation et la diffusion des connaissances. Il nous revient, aujourd'hui, de faire parler ces objets, qu'il s'agisse d'outils, d'instruments scientifiques, de véhicules ou de jouets, qu'ils aient dix siècles, dix ans ou dix jours.

\section{Bibliographie}

[BLA 16] BLANCHARD A., " Montrer la science en train de se faire ? Du Palais de la découverte à la sociologie des sciences », Alliage, n 77, p. 50-59, 2016.

[BOI 72] BOIREL R., L'Invention, PUF, Paris, 1972.

[DAU 79] DAUMAS M., (dir.), Histoire générale des techniques, PUF, Paris, 1979.

[DEF 85] DEFORGE Y., Technologie et génétique de l'objet industriel, Maloine, Paris, 1985.

[EDG 13] EDGERTON D., Quoi de neuf ? : du rôle des techniques dans l'histoire globale, Seuil, L'Univers historique, Paris, 2013.

[FER 97] FERRIOT D., JACOMY B., «Problématique d'une rénovation: Musée des arts et métiers », dans SCHIELE B., et KOSTER E. (dir.), La révolution de la muséologie des sciences: vers les musées du XXI siècle, Presses Universitaires de Lyon, Lyon,1997.

[GIL 78] GILLE B., Histoire des techniques, Gallimard, Encyclopédie de la Pléiade, Paris, 1978.

[GIM 75] GIMPEL J., La Révolution industrielle du Moyen Âge, Éd. du Seuil, Paris, 1975.

[GIM 92] GIMPEL J., La fin de l'avenir : la technologie et le déclin de l'occident, Éd. du Seuil, Paris, 1992.

[HAU 88] HAUDRICOURT A-G., La technologie science humaine : recherches d'histoire et d'ethnologie des techniques, Maison des sciences de l'homme, Paris, 1988.

[JAC 90] JACOMY B., Une histoire des techniques, Éd. du Seuil, Points Sciences, Paris, 1990.

[JAC 93] JACOMY B., « Culture technique de l'ingénieur », Les Techniques de l'ingénieur, nº T 40, 1993.

[JAC 98] JACOMY B., "The Technological Evolution of Riveting Machines ». IA, the Journal of the Society for Industrial Archeology, 24, nº 2, p. 37-52, 1998.

[JAC 15] JACOMY B., Une histoire des techniques, Éd. du Seuil, Points Sciences, Paris, 2015.

[KUH 72] KUHN T., La structure des révolutions scientifiques, Flammarion, Paris, 1972.

[LAT 14] LATOUR B., " L'Anthropocène et la destruction de l'image du Globe », dans HACHE E. (Dir.), De l'univers clos au monde infini, p. 30, Éd. Dehors, Paris, 2014.

[LEB 03] LEBEAUME, J., «Vers la technologie et la culture technique pour tous. Quelques repères du passé pour penser les défis de demain » dans Livre des résumés et contributions du colloque Culture technique : enjeu de société, IRHESCFSU, Paris, p. 29-38, 2003.

[LEC 15] LECUYER C., « Manager l'innovation », dans BONNEUIL C., PESTRE D. (dir.), Histoire des sciences et des savoirs. Tome 3 : Le siècle des technosciences, Éd. du Seuil, Paris, 2015.

[LER 45] LEROI-GOURHAN A., Évolution et techniques, «L'homme et la matière », t. 1, Albin Michel, Paris, 2 vol., 1945.

[MIN 15] MINISTERE DE L'ÉDUCATION NATIONALE, DE L'ENSEIGNEMENT SUPERIEUR ET DE LA RECHERCHE, Programmes pour les cycles 2, 3, 4, 2015.

[MOL 70] MOLES A., CAUDE R., Créativité et méthodes d'innovation dans l'entreprise, Fayard-Mame, Paris, 1970.

[MOL 72] MOLES A., Théorie des objets, Éd. Universitaires, Paris, 1972.

[MOR 16] MORIN E., « Changer de voie », Le 1, 17 février 2016. 
[ROQ 83] ROQUEPLO P., Penser la technique, Éd. du Seuil, Paris, 1983.

[SIG 91] SIGAUT F., « Aperçus sur l'histoire de la technologie en tant que science humaine », dans J-P. BOMPARD ET J. PERRIN (Dir.), Histoire des techniques et compréhension de l'innovation : séminaire de recherche, mars 1989-février 1990, Institut national de la recherche agronomique, Économie et sociologie rurales, "Actes et communications », Paris, 1991.

[SIM 89] SIMONDON G., Du Mode d'existence des objets techniques, Aubier-Montaigne, Analyse et raisons, Paris, 1989.

[VER 93] VERIN H., La Gloire des ingénieurs, l'intelligence technique du XVI au XVIII esiècle, Albin Michel, Paris, 1993.

[WHI 69] WHITE L. JR., Technologie médiévale et transformations sociales, Mouton, Paris, La Haye, p. 1-53, 1969. 